ResearchArticle

\title{
Energy Dissipation-Based Method for Strength Determination of Rock under Uniaxial Compression
}

\author{
M. M. He ${ }^{10},{ }^{1,2}$ F. Pang, ${ }^{2}$ H. T. Wang, ${ }^{2}$ J. W. Zhu, ${ }^{3}$ and Y. S. Chen ${ }^{2}$ \\ ${ }^{1}$ State Key Laboratory of Eco-Hydraulics in Northwest Arid Region, Xi'an University of Technology, Xi'an 710048, China \\ ${ }^{2}$ Shaanxi Key Laboratory of Loess Mechanics and Engineering, Xi'an University of Technology, Shaanxi 710048, China \\ ${ }^{3} X i$ 'an Research Institute of China Coal Research Institute, Xi'an 710077, China
}

Correspondence should be addressed to M. M. He; 807658619@qq.com

Received 1 May 2020; Revised 28 July 2020; Accepted 31 July 2020; Published 13 August 2020

Academic Editor: Fabio Rizzo

Copyright (C) 2020 M. M. He et al. This is an open access article distributed under the Creative Commons Attribution License, which permits unrestricted use, distribution, and reproduction in any medium, provided the original work is properly cited.

\begin{abstract}
The energy conversion in rocks has an important significance for evaluation of the stability and safety of rock engineering. In this paper, some uniaxial compression tests for fifteen different rocks were performed. The evolution characteristics of the total energy, elastic energy, and dissipated energy for the fifteen rocks were studied. The dissipation energy coefficient was introduced to study the evolution characteristics of rock. The evolution of the dissipation energy coefficient for different rocks was investigated. The linear interrelations of the dissipation energy coefficients and the yield strength and peak strength were explored. The method was proposed to determine the strength of rock using the dissipation energy coefficients. The results show that the evolution of the dissipation energy coefficient exhibits significant deformation properties of rock. The dissipation energy coefficients linearly increase with the compaction strength, but decrease with the yield strength and peak strength. Moreover, the dissipation energy coefficient can be used to determine the rock burst proneness and crack propagation in rocks.
\end{abstract}

\section{Introduction}

Geotechnical engineering problems in civil, mining, and petroleum engineering practices [1]: the mechanical properties of rock have become the most common measurement in most rock mass classification systems [2,3]. In the field of underground engineering, rocks undergo the complex energy change, which includes energy input, accumulation, dissipation, and release $[4,5]$. Consequently, the study of energy conversion in rock under the loading process has a significant influence on the stability and safety analyses in rock engineering projects.

The deformation and failure process of rock is the gradual damage evolution process driven by energy. It would be better to describe the deformation and failure of rocks from the perspective of energy [6]. Some researchers have carried out experimental studies and numerical simulations on the variation of energy in the deformation and failure process of rocks. Based on numerical simulations, Ju et al. [7] built a three-dimensional finite element model of the porous rock-like medium and investigated mechanisms of deformation and failure and energy dissipation rule of porous rock-like media. Joints play an important role in the mechanical behavior of rock mass, and the mechanical behavior of joints changes with the change of scale. Wang et al. [8] studied the effect of scale on the mechanical properties of rock by means of numerical simulation. Meng et al. [9] experimentally studied the characteristics of energy in uniaxial cyclic loading and unloading compression of sandstone rock under six different loading rates and proposed that the energy evolution of rock is closely related to the axial loading stress, rather than the axial loading rate. Wang and Cui [10] revealed the relation between energy change and confining pressure during the process of sandstone damage and characteristics of energy storage and energy dissipation in different deformation stage. Huang and $\mathrm{Li}$ [11] found that the magnitude of initial confining pressure and the unloading rate significantly influence rock strain energy conversion during unloading. The effects of water content on the energy evolution of red sandstone have 
also been investigated. The experimental results indicate that the saturation process greatly reduces the magnitude and rate of energy release [12]. Ma et al. [13] studied the energy dissipation mechanism of the rock deformation process under the influence of freeze-thaw cycles. Li et al. [14] established a damage model for the fractured rock based on energy dissipation. According to the full stress-strain curve of rock, a new evaluation method of rock brittleness is put forward on the basis of energy evolution $[15,16]$. Chen et al. [17] carried out uniaxial compression tests on coal-rock composite specimens. The strength, macroscopic failure initiation, and failure characteristics of coal-rock composite specimens were studied. The abovementioned researches mainly focus on characteristics of energy under different conditions or concentrate on a specific energy (strain energy). However, a few studies have emphasized the relationship between energy and strength of rock during the rock deformation and failure process $[18,19]$.

In this work, the uniaxial compression tests were conducted for different rocks to study the energy conversion. The evolution of the total input energy, elastic energy, and dissipation energy was systematically analyzed during the deformation and failure process of rock. A new energy parameter, dissipation energy coefficient, was introduced. The purpose is to clarify the energy evolution characteristics. Besides, the linear relationship between the dissipation energy coefficient and the strength of rock was also established.

\section{Preparation of the Sample and Experimental Methodology}

2.1. Preparation of the Sample. The rock specimens in this experiment were mudstone, physicochemical slate, schist, limestone, gneiss, sandstone, porphyrite, dolomite, shale, metamorphic sandstone, marble, quartz schist, quartzite, diorite, and granite. They were collected from a quarry in Shaanxi, China. All specimens were drilled and processed into $\varphi 50 \mathrm{~mm} \times 100 \mathrm{~mm}$ (diameter $\times$ height $)$ standard cylinders. The nonparallel between the two end faces of the samples was $<0.005 \mathrm{~mm}$. The measurement error was within $0.3 \mathrm{~mm}$, when the diameter was measured along the height of the samples [20]. Table 1 presents the basic parameters of the test samples.

2.2. Experimental Apparatus and Scheme. The uniaxial compression tests were conducted on the WDT-1500 testing system (Figure 1), which consists of four parts: the loading system, measuring system, power system, and control system $[21,22]$. The WDT test system can provide the axial loading capacity of $1500 \mathrm{kN}$, confining pressure of $80 \mathrm{MPa}$, and frequency of $10 \mathrm{~Hz}$. The WDT-1500 multifunctional material testing machine used in this paper has a large rigidity. The control system adopts a DOLI full digital servo controller imported from Germany, which has the characteristics of high precision and stability. It can control not only the load but also the displacement and strain and can obtain a good rock full stress-strain curve. At the beginning of each test, the initial load is $1 \mathrm{kN}$, which ensures full contact between the sample and the compression plate.
Then, the loading rate was set to $0.5 \mathrm{~mm} / \mathrm{min}$, and the loading was applied continuously until the failure occurred. During the test, the data collection interval was $0.2 \mathrm{~s}$.

2.3. Calculation of Energy. It is assumed that the deformation and failure process of rock under the external load is no heat loss [23]. The total energy can be calculated according to the first law of thermodynamics as follows:

$$
U=U^{e}+U^{d}
$$

where $U$ is the total energy, $U^{e}$ is the elastic energy, and $U^{d}$ is the dissipated energy. The elastic energy generally stored in rock and the dissipated energy caused the plastic deformation and crack propagation in the specimen. Figure 2 illustrates the relationship between the elastic energy and the dissipated energy of rock elements under uniaxial compression conditions [24]. As shown in Figure 2, the area of the shadow represents the elastic strain energy, and the red area represents the dissipated energy. Each energy was calculated as follows [25]:

$$
\begin{aligned}
U & =\int_{0}^{\varepsilon_{1}} \sigma \mathrm{d} \varepsilon_{1}, \\
U^{e} & =\frac{\sigma^{2}}{2 E}, \\
U^{d} & =U-U^{e},
\end{aligned}
$$

where $\sigma$ and $\varepsilon_{1}$ are axial stress and strain, respectively.

The deformation and failure process of rock involves the complex energy conversion. To analyze the process of the energy evolution, the dissipation energy coefficient was introduced, which is the ratio of the dissipated energy to the elastic energy at any time during the deformation process of rock [26]:

$$
\begin{aligned}
& \lambda=\frac{U^{d}}{U^{e}}, \\
& \lambda_{v}=\frac{U_{v}^{d}}{U_{v}^{e}},
\end{aligned}
$$

where $U_{v}^{d}, U_{v}^{e}$, and $\lambda_{v}$ are the dissipated energy, elastic energy, and dissipated energy coefficient of the compaction point, respectively.

$$
\lambda_{u}=\frac{U_{u}^{d}}{U_{u}^{e}}
$$

where $U_{u}^{d}, U_{u}^{e}$, and $\lambda_{u}$ are the dissipated energy, elastic energy, and dissipated energy coefficient of the yield point, respectively.

$$
\lambda_{c}=\frac{U_{c}^{d}}{U_{c}^{e}},
$$

where $U_{c}^{d}, U_{c}^{e}$, and $\lambda_{c}$ are the dissipated energy, elastic energy, and dissipated energy coefficient of the yield point, respectively. $\lambda_{v}, \lambda_{u}$, and $\lambda_{c}$ are the dissipation energy coefficient at three different feature points, respectively. They 
TABLE 1: Rock types and basic parameters.

\begin{tabular}{|c|c|c|c|c|c|c|c|}
\hline Rock types & $\begin{array}{c}\text { Density } \\
\rho\left(\mathrm{g} / \mathrm{cm}^{3}\right)\end{array}$ & $\begin{array}{c}\text { Moisture } \\
\text { content } \\
\omega(\%)\end{array}$ & $\begin{array}{c}\text { Water } \\
\text { absorption } \\
\omega_{a}(\%)\end{array}$ & $\begin{array}{c}\text { Compressive } \\
\text { strength } \\
\sigma_{c}(\mathrm{MPa})\end{array}$ & $\begin{array}{c}\text { Elastic } \\
\text { modulus } \\
E(\mathrm{GPa})\end{array}$ & $\begin{array}{c}\text { Tensile } \\
\text { strength } \\
\sigma_{t}(\mathrm{MPa})\end{array}$ & $\begin{array}{c}\text { Poisson's } \\
\text { ratio } \\
u\end{array}$ \\
\hline Mudstone & 2.50 & 2.36 & 3.30 & 32.53 & 4.21 & 4.37 & 0.24 \\
\hline Physicochemical slate & 2.72 & 0.87 & 1.28 & 32.48 & 4.06 & 4.28 & 0.24 \\
\hline Schist & 2.75 & 0.36 & 0.50 & 41.11 & 4.53 & 4.75 & 0.23 \\
\hline Limestone & 2.63 & 0.29 & 0.47 & 37.15 & 4.72 & 4.32 & 0.24 \\
\hline Gneiss & 2.72 & 0.22 & 0.74 & 38.52 & 4.81 & 4.25 & 0.24 \\
\hline Sandstone & 2.67 & 1.89 & 2.73 & 55.64 & 6.75 & 6.24 & 0.23 \\
\hline Porphyrite & 2.60 & 1.57 & 2.35 & 65.77 & 7.46 & 6.87 & 0.22 \\
\hline Dolomites & 2.70 & 0.35 & 0.71 & 91.57 & 10.35 & 8.79 & 0.21 \\
\hline Shale & 2.68 & 0.52 & 1.12 & 90.03 & 9.78 & 8.63 & 0.20 \\
\hline $\begin{array}{l}\text { Metamorphic } \\
\text { sandstone }\end{array}$ & 2.74 & 0.03 & 0.73 & 99.66 & 11.64 & 9.73 & 0.20 \\
\hline Marble & 2.68 & 0.04 & 0.75 & 105.95 & 12.25 & 10.54 & 0.19 \\
\hline Quartz schist & 2.76 & 0.14 & 0.72 & 127.03 & 14.95 & 11.83 & 0.19 \\
\hline Quartzite & 2.81 & 0.08 & 0.70 & 164.36 & 19.29 & 12.56 & 0.18 \\
\hline Diorite & 2.80 & 0.11 & 0.71 & 207.44 & 24.75 & 14.28 & 0.17 \\
\hline Granite & 2.85 & 0.05 & 0.35 & 261.58 & 29.65 & 15.36 & 0.16 \\
\hline
\end{tabular}

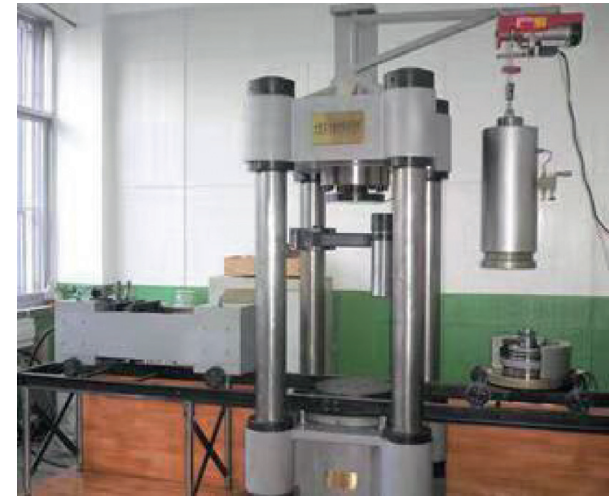

Figure 1: The WDT-1500 multifunctional material testing machine.

reflect the changes in the primary and secondary status of elastic energy and dissipated energy during the process of deformation and failure of rock, respectively.

\section{Test Result}

3.1. Stress-Strain Curves Characteristics. The deformation and failure process of rock has four stages: the compaction stage, elastic deformation stage, yield stage, and failure stage [27].

(1) Compaction stage: the stress-strain curve is nonlinear, which results from the closure of some primary microcracks and pores under the action of initial pressure [28].

(2) Elastic deformation stage: the stress-strain curve is linear, and the slope of linear portions is the elastic modulus.

(3) Yield stage: as the stress increases, the stress-strain curves gradually depart from the linear. It is due to the fact that the sample gradually transformed from elastic deformation to elastic-plastic deformation.

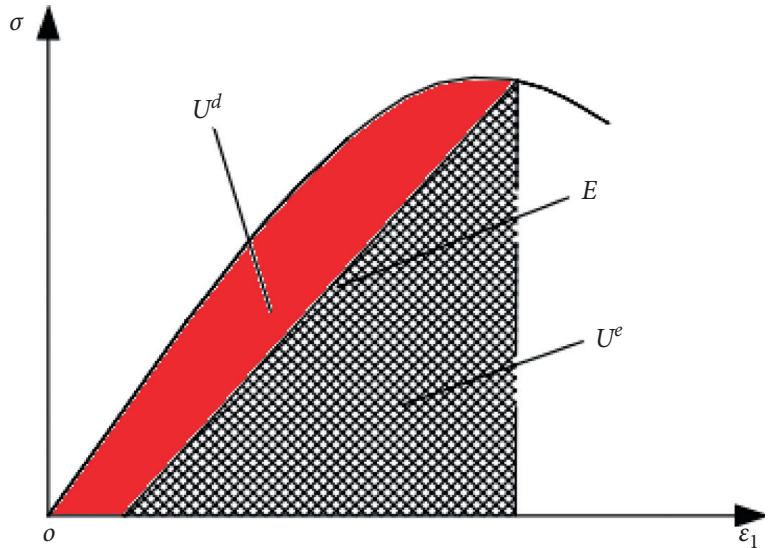

FIGURE 2: Relationship between the elastic strain energy and dissipated energy of rock under uniaxial compression [24].

(4) Failure stage: when the peak strength is reached, the sample shows macroscopic fracture. Moreover, the postpeak curves present two different failure characteristics (Figure 3). One shows an apparent brittle failure characteristic (such as granite, diorite, quartzite, limestone, gneiss, dolomite, shale, metamorphic sandstone, and quartz schist), and the other shows an obvious ductile failure characteristic (such as mudstone, physicochemical slate, schist, porphyrite, sandstone, and marble) $[29,30]$.

3.2. Characteristics of Total Energy. The deformation and failure process of rock also is the process of the generation, expansion, connection, and slip of the microcracks. The generation of new cracks needs absorption of energy, and the friction between the crack surfaces dissipate energy. Actually, the process of rock deformation and failure is the process of energy accumulation and dissipation [31]. 


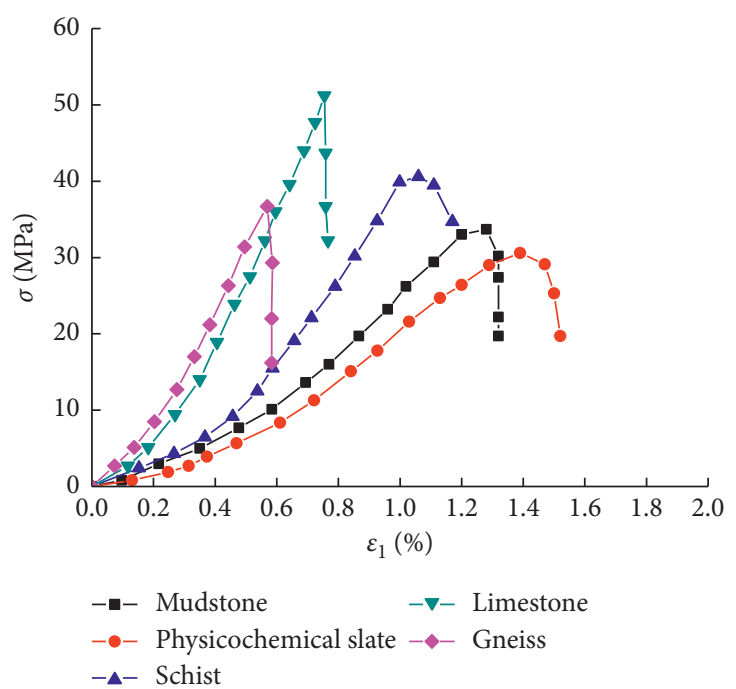

(a)

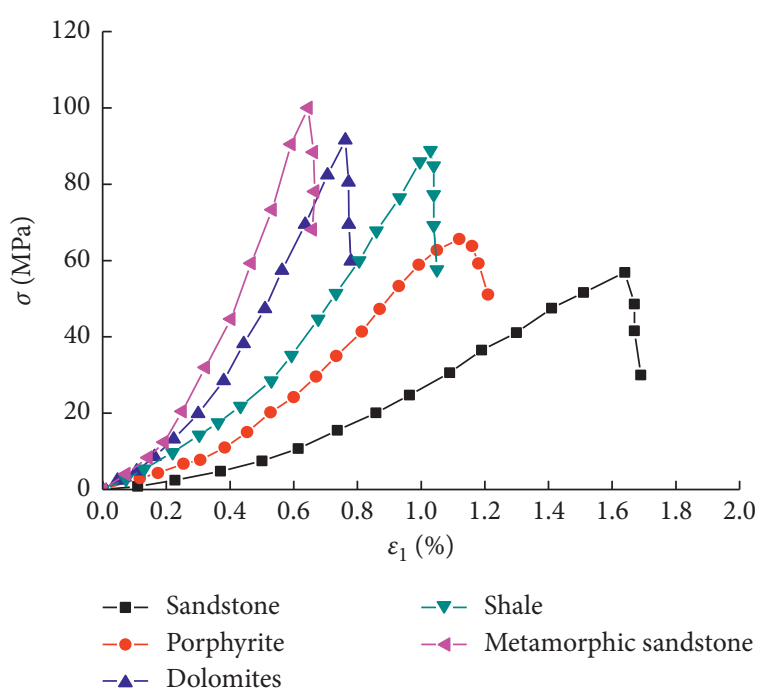

(b)

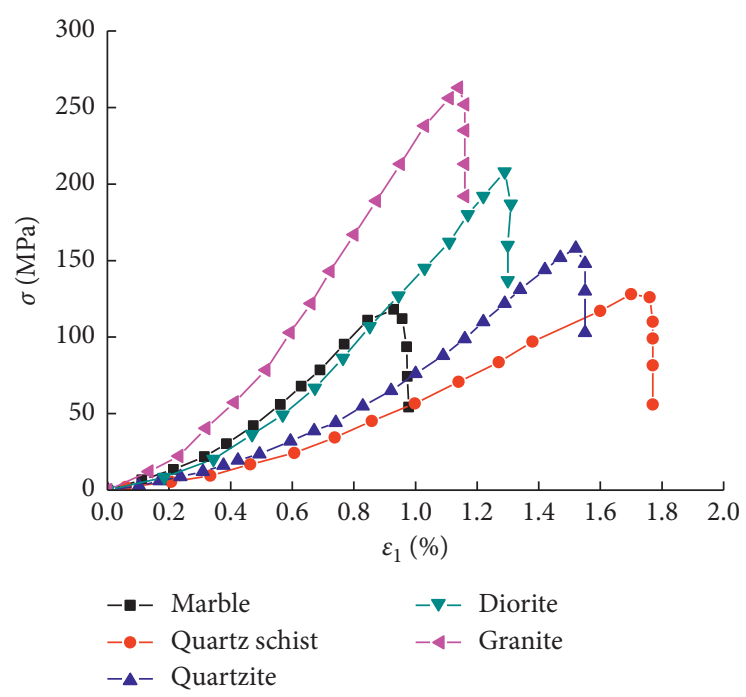

(c)

FIGURE 3: Stress-strain curves for different rocks under the uniaxial compression condition.

Figure 4 illustrates the relationships between the total energy $(U)$ and axial stress $(\sigma)$ for different rocks under uniaxial tests. As shown in Figure 4, the total energy nonlinearly increases with the applied stress in the initial compaction stage. In the elastic deformation stage, the total energy linearly increases with the increase in stress, and the growth rate obviously seems to remain constant. After entering the yield stage, the total energy continues to increase with the stress, but the growth rate gradually decreases. In the failure stage, the total energy shows two different trends, which results from 15 different rocks with two different types of failure characteristics $[32,33]$.

3.3. Characteristics of Elastic Energy. Figure 5 depicts the relationship between the elastic energy and stress for different rocks under the uniaxial compression condition. The elastic energy hardly increases in the microcracks compaction stage. The reason is that the closure of internal cracks and pores dissipates a great number of energy, and only a small amount of energy is converted into the elastic energy. The elastic energy of gneiss and sandstone is around $15 \mathrm{~kJ} /$ $\mathrm{m}^{3}$ and $20 \mathrm{~kJ} / \mathrm{m}^{3}$, respectively (Figures $5(\mathrm{a})$ and $5(\mathrm{~b})$ ). The elastic energy increases linearly with the stress, and the growth rate reaches the maximum in the elastic stage. It was due to a stable elastic deformation generated in the sample. It makes a large amount of the energy transformation into the elastic energy. After entering the yield stage, the cracks in rock began to develop and expand. The elastic energy continues to increase with the stress, but the growth rate of the elastic energy gradually decreases. The elastic energy reaches the maximum value (the storage limit value) at the peak point, and the maximum elastic energy of shale and marble is around $320 \mathrm{~kJ} / \mathrm{m}^{3}$ and $430 \mathrm{~kJ} / \mathrm{m}^{3}$, respectively (Figures 5(b) and 5(c)). In the failure stage, the elastic energy accumulated in the sample is released rapidly, which makes the internal cracks of the sample expand rapidly and the sample failed [34]. 


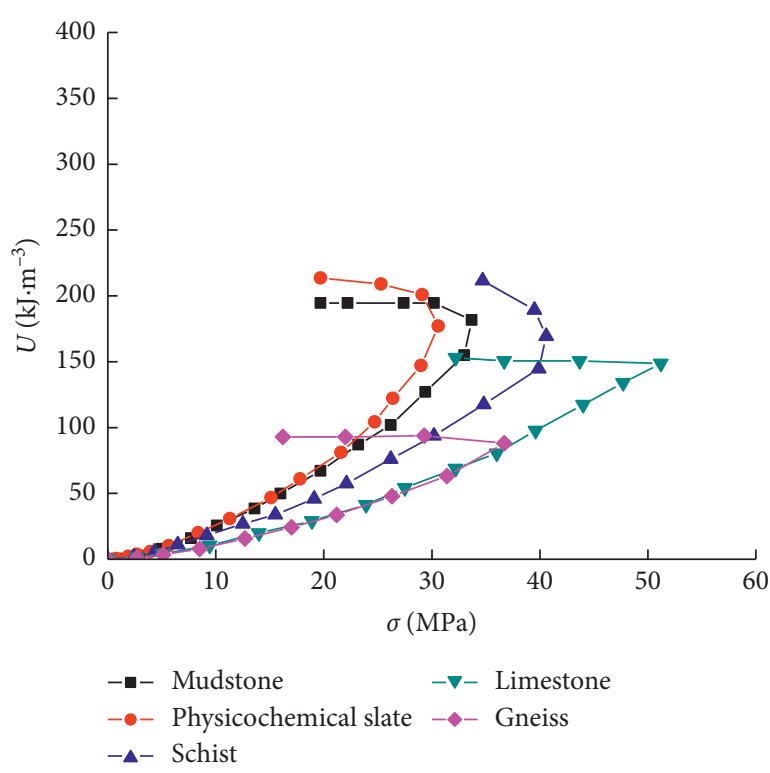

(a)

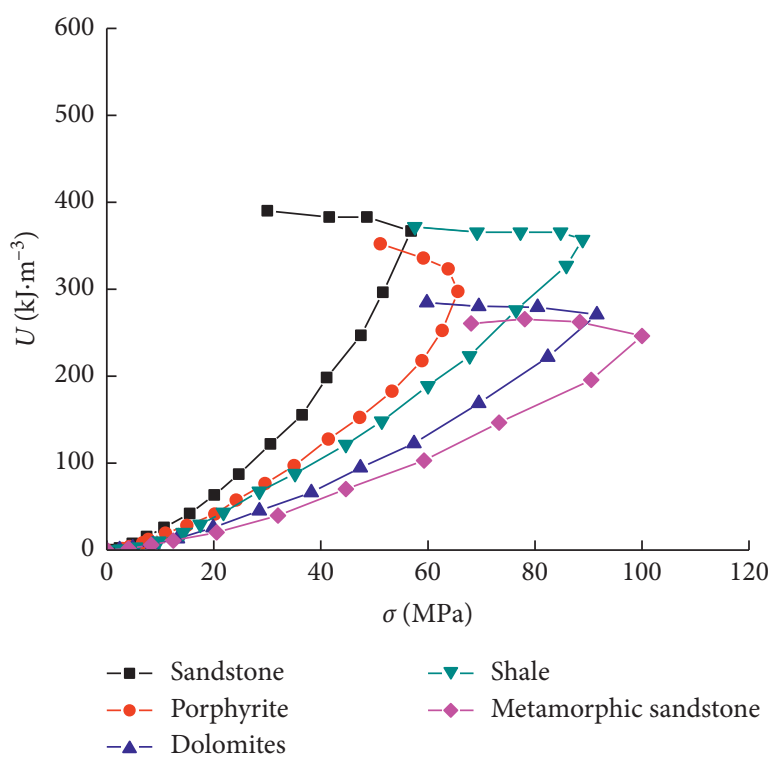

(b)

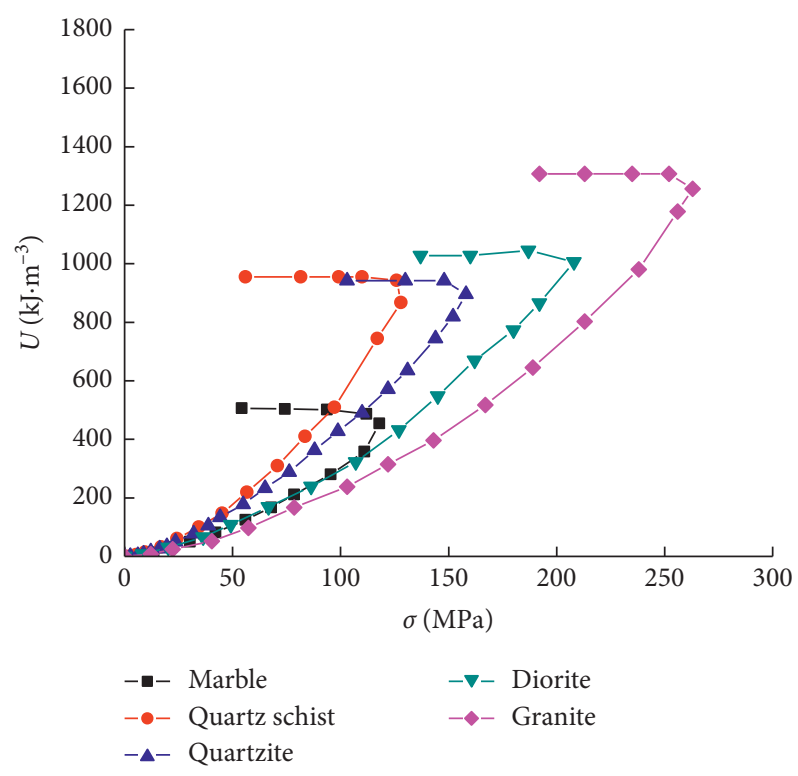

(c)

Figure 4: Variation of total energy with stress for different rocks.

3.4. Characteristics of Dissipated Energy. The energy dissipation is the main factor leading to the internal damage of rocks [35]. Figure 6 shows variation of the dissipated energy with stress for different rocks. The dissipated energy rapidly increases in the compaction stage. The reason is that more energy is consumed for the closed friction of the initial cracks and pores in rocks and the creation of new cracks in the structural redistribution. In the elastic stage, the growth rate of the dissipated energy is gradually reduced, and the dissipated energy is relatively low. This was due to the generated recoverable elastic limit by the sample, and it hardly produced new microcracks and internal friction. The total energy was basically converted into elastic energy, and the dissipated energy was maintained at a stable level $[36,37]$. The values of limestone and metamorphic sandstone are stabilized at around $10 \mathrm{~kJ} / \mathrm{m}^{3}$ and $15 \mathrm{~kJ} / \mathrm{m}^{3}$, respectively (Figures $6(\mathrm{a})$ and 6(b)). After entering the yield stage, the dissipated energy starts to steadily increase, and the growth rate gradually increases. This is because the rock deformation transformed from linear elasticity into nonlinear elasticity, and the microcracks inside the rock began to expand rapidly and generate new cracks. In the failure stage, the dissipated energy sharply increases and exceeds the elastic energy in the end.

3.5. Characteristics of the Dissipation Energy Coefficient. The dissipation energy coefficient $\lambda$ is defined as the ratio of the dissipated energy to the elastic energy. Figure 7 shows the variation of the dissipation energy coefficient with stress for different rocks. The dissipation energy coefficient has four 


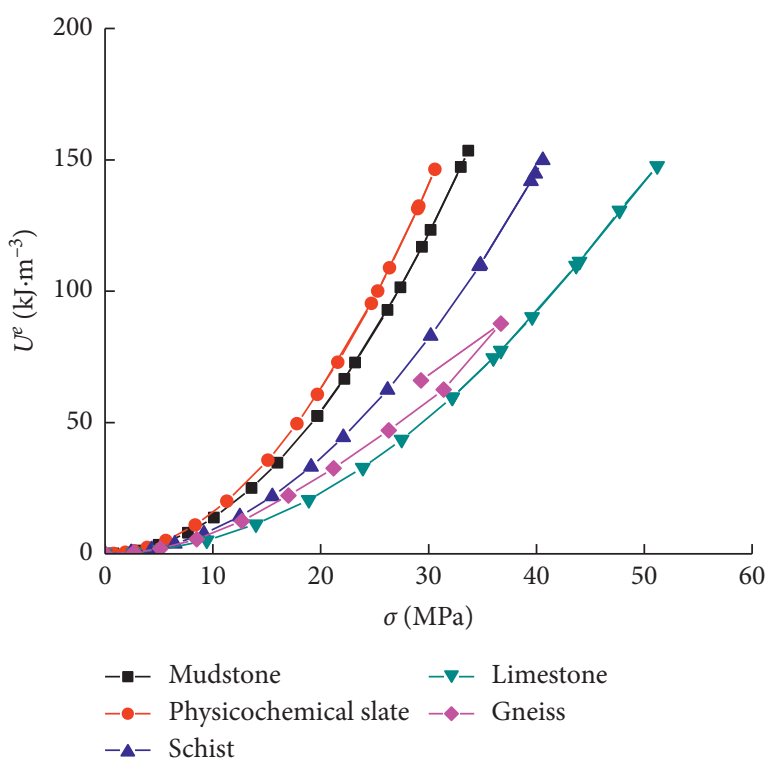

(a)

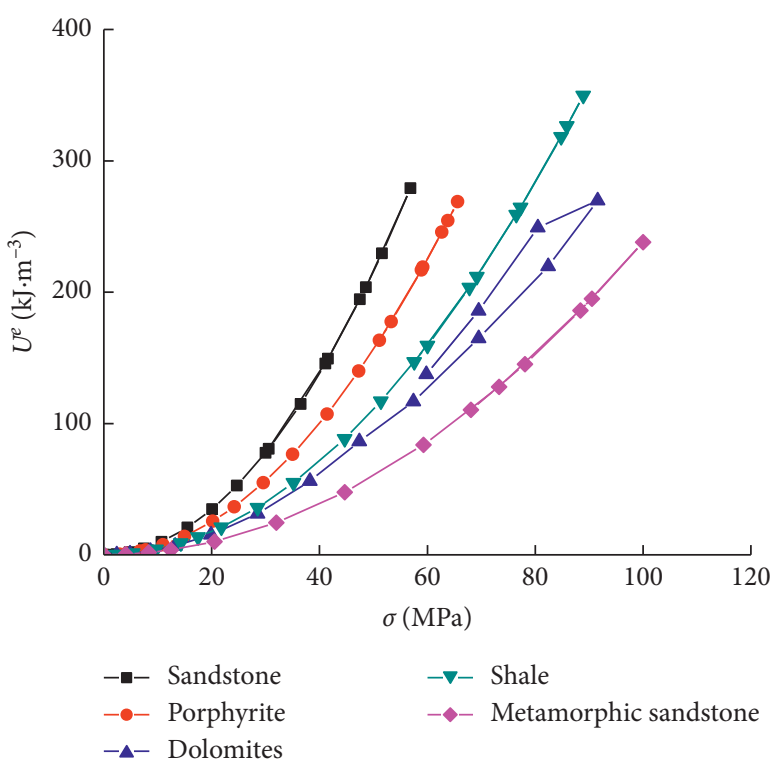

(b)

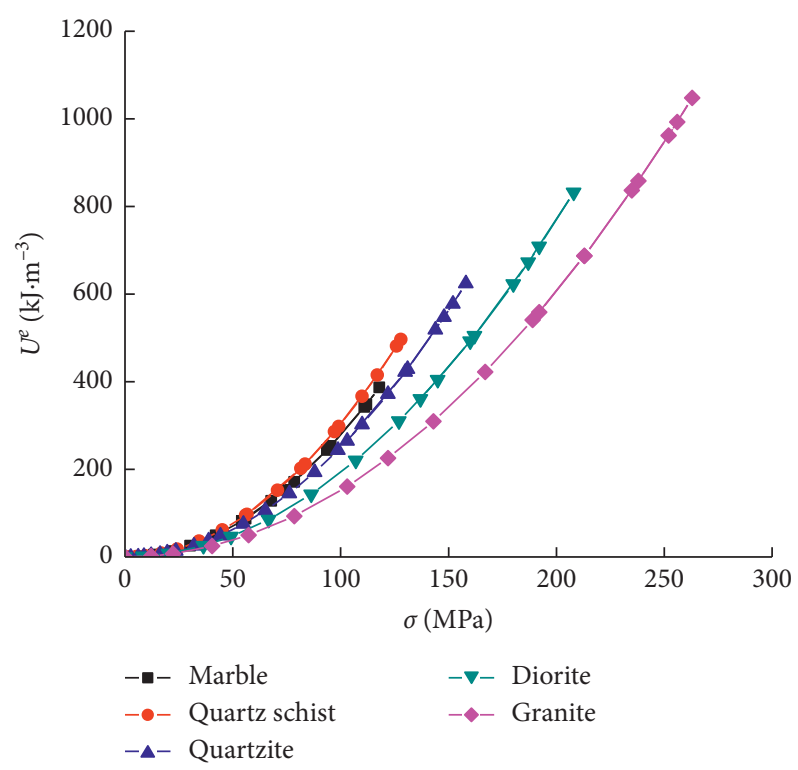

(c)

FIgURE 5: Variation of elastic energy with stress for different rocks.

evolution stages and three distinct characteristic points (Figure $7(\mathrm{~d})$ ).

The compaction stage (OA): the point $\mathrm{A}$ is called the compaction point, which also is the first characteristic point of the curve. The stress of point $\mathrm{A}$ is defined as compaction strength. The dissipation energy coefficient $\lambda$ rapidly increases with stress. It is due to the fact that the closure and friction of the microcrack in the rock consume most of the energy during the initial compaction stage, and only an extremely small amount of energy was converted into the elastic energy. Therefore, the dissipation energy coefficient $\lambda$ rapidly increases with stress.

The elastic deformation stage $(A B)$ : the point $B$ is called the yield point, which is the second characteristic point of the curve. The dissipation energy coefficient $\lambda$ decreases as the stress increases and reaches the minimum value at the yield point $\mathrm{B}$. The reason is the microcracks in the rock are completely closed at this stage, and the total energy is basically converted into the elastic energy. Moreover, there is almost no generation or propagation of new cracks at this stage, and the energy dissipation is relatively low. Therefore, the dissipation energy coefficient $\lambda$ decreases.

The yield stage (BC): the point $C$ is called the peak point, which is the third characteristic point of the curve. The dissipation energy coefficient $\lambda$ slowly increases as the stress increases. It is due to the new microcracks gradually generated inside the rock. Moreover, the number of microcracks increases with increase in stress. This result in a rapid growth in dissipated energy and the slow growth of the elastic 


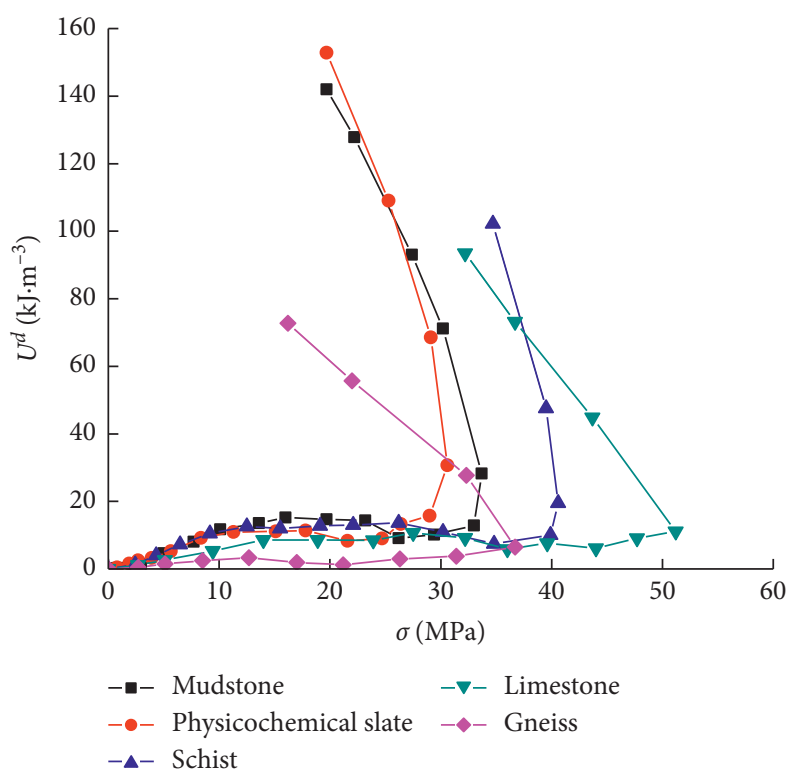

(a)

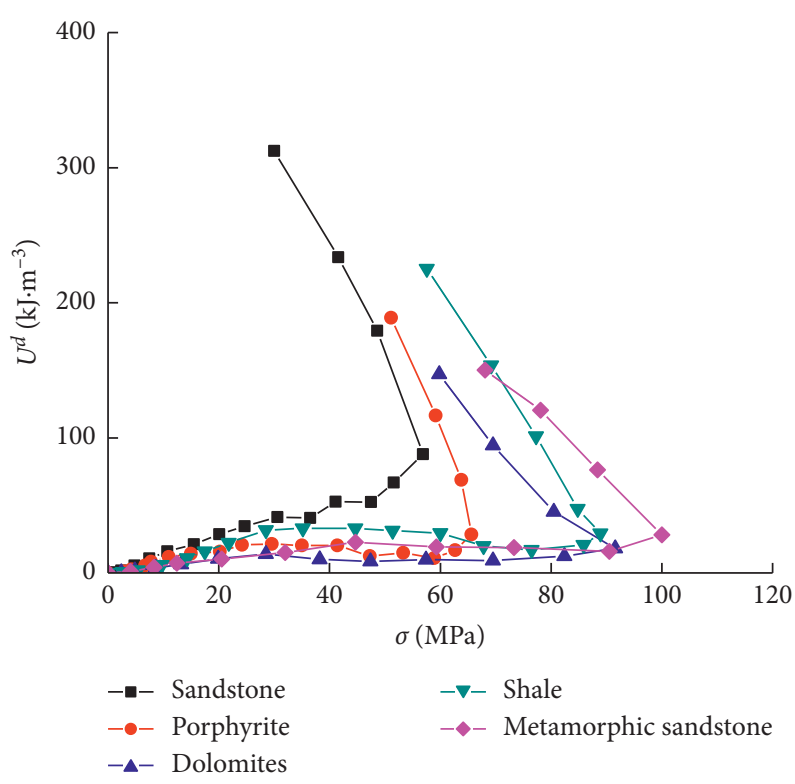

(b)

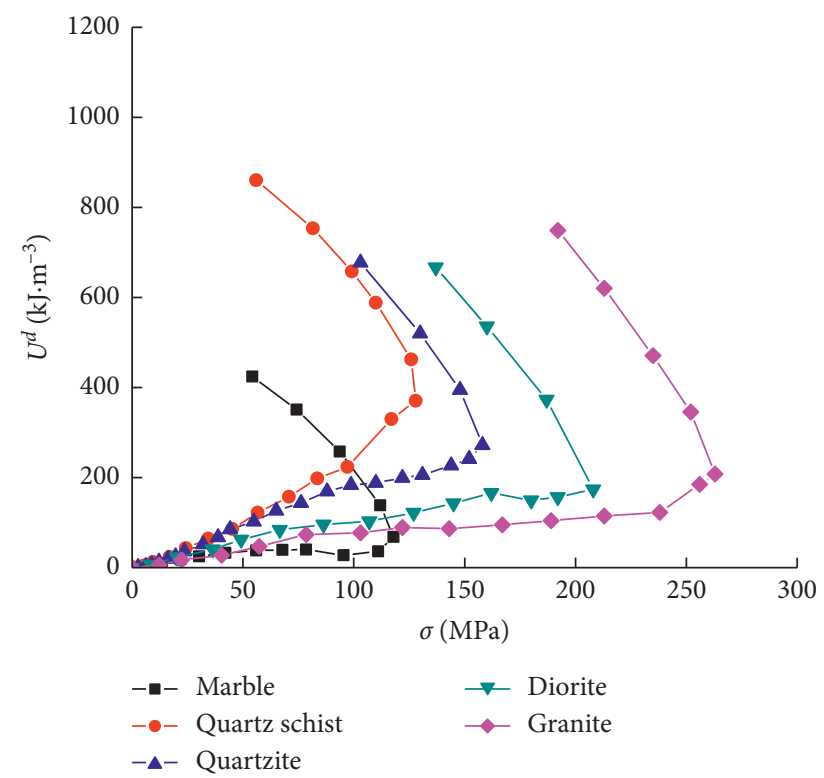

(c)

FIGURE 6: Variation of dissipated energy with stress for different rocks.

energy. Thence, the dissipation energy coefficient $\lambda$ slowly increases as the stress increases.

The failure stage $(\mathrm{CD})$ : the dissipation energy coefficient $\lambda$ dramatically increases. The reason lies in the following two aspects. On the one hand, the generation of the macroscopic cracks makes the elastic energy to be released continuously. On the other hand, the cracks propagation is accelerated and the relative slip between the particles becomes larger, which make the dissipated energy to increase rapidly.

It can also be seen that the primary and secondary status of elastic energy and dissipated energy vary during the four stages. In the initial stage of loading, $U^{e}$ and $U^{d}$ increase slowly, but the increment of $U^{d}$ is greater than the increment of $U^{e} . U^{d}$ dominates at this stage. In the elastic stage, $U^{e}$ and $U^{d}$ increase, and the increment in $U^{d}$ is smaller than that of $U^{e}$. $U^{e}$ predominates in the energy conversion process. In the plastic deformation stage, $U^{d}$ increases rapidly, while $U^{e}$ decreases. The dominant one is still elastic energy. In the failure stage, the elastic energy stored in the rock is almost converted into dissipated energy. At this stage, the dissipated energy plays an important role, which causes macroscopic damage and strength loss of the sample.

According to Table 2, the relationships between the dissipation energy coefficients and strength at three different characteristic points are studied (Figure 8). The results show that the dissipation energy coefficient linearly 


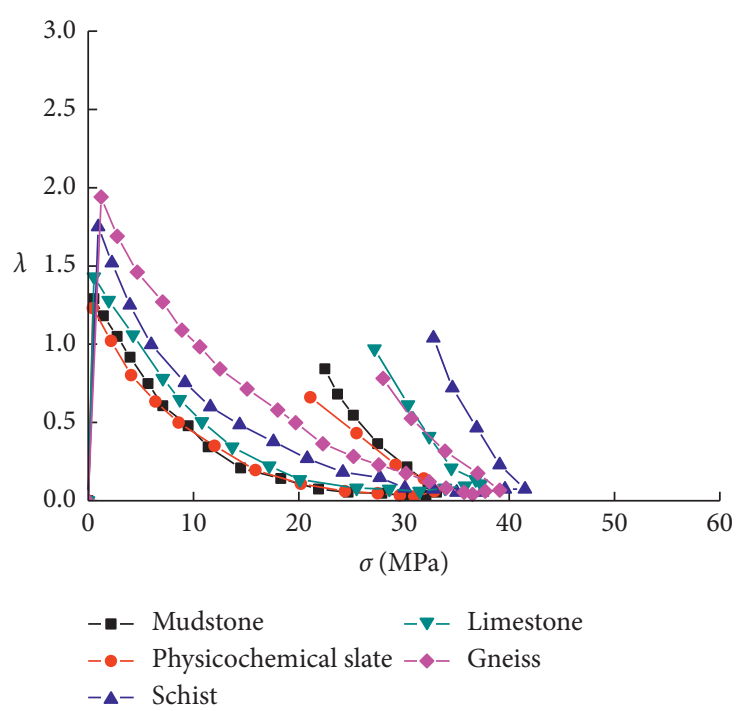

(a)

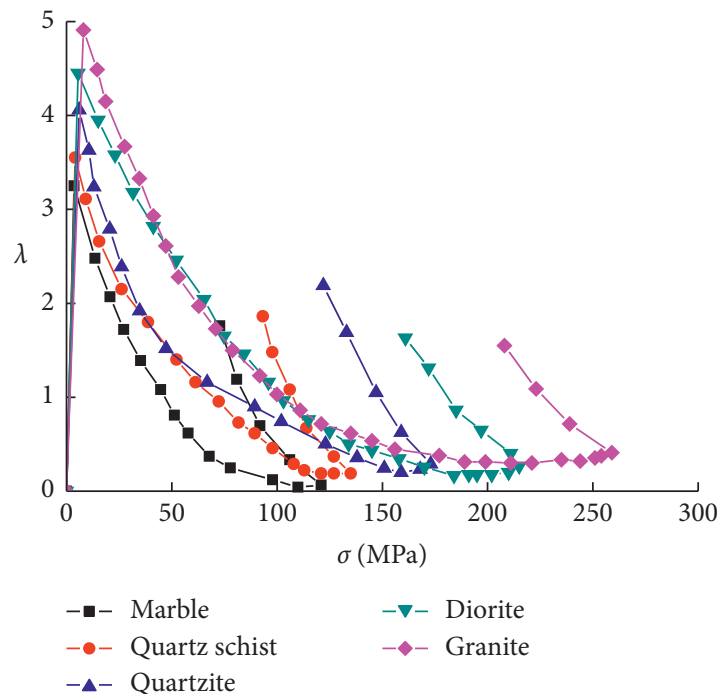

(c)

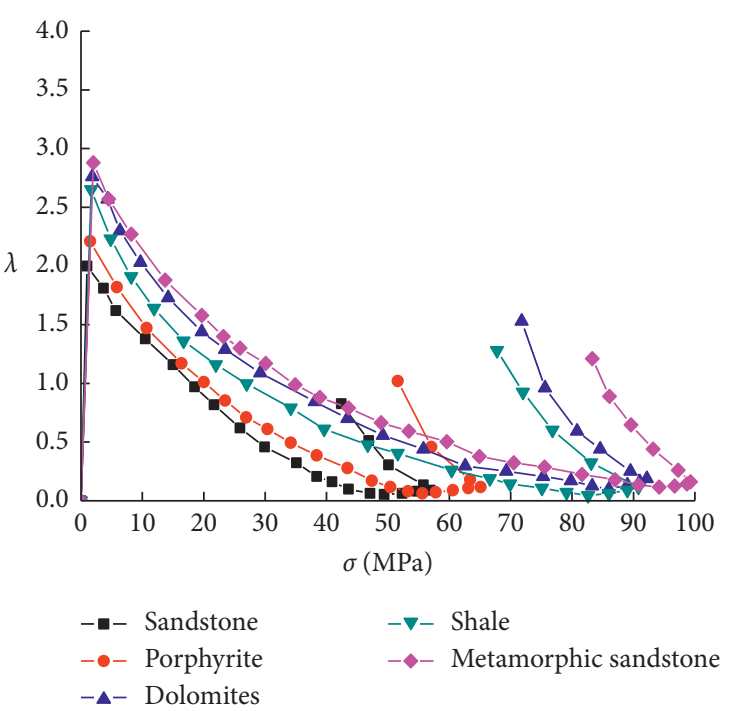

(b)

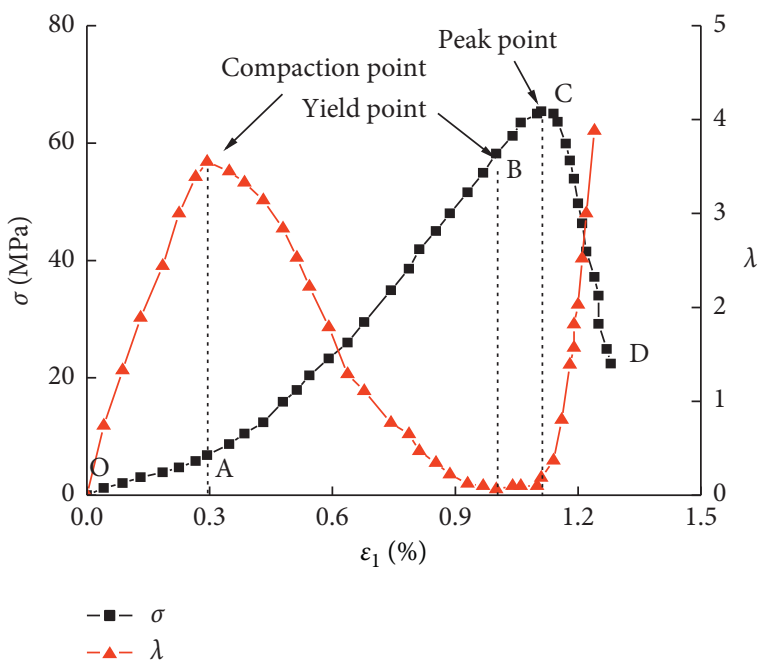

(d)

Figure 7: Variation of the dissipation energy coefficient with stress for different rocks.

increases with an increase in compaction strength (Figure 8(a)). However, the dissipation energy coefficient linearly decreases with yield strength and peak strength (Figures $8(\mathrm{~b})$ and $8(\mathrm{c})$ ). The relationships can be expressed with the linear fitting functions. Figure 8 also lists the fitting functions and the correlation coefficient $\left(R^{2}\right)$, respectively. The correlation coefficient $R^{2}$ is $0.97,0.94$, and 0.94 , respectively. It shows that there is a strong linear relationship between the dissipation energy coefficients and strength in three different characteristic points.

$\sigma_{v}, \sigma_{u}$, and $\sigma_{c}$ are defined as compaction strength, yield strength, and peak strength, respectively. $\left(\sigma_{u} / \sigma_{c}\right)$ is defined as the characteristic stress, and $\left(\lambda_{u} / \lambda_{c}\right)$ are defined as the characteristic dissipation energy coefficients. The relationship between the characteristic stress and the characteristic dissipation energy coefficient was studied (Figure 9). The characteristic dissipation energy coefficient linearly increases with the characteristic stress. The fitting function is expressed as follows:

$$
\frac{\lambda_{u}}{\lambda_{c}}=1.43782 \frac{\sigma_{u}}{\sigma_{c}}-0.64269 .
$$

The correlation coefficient $R^{2}$ of the fitting functions is 0.91. It shows there is a strong linear relationship between the characteristic stress and the characteristic dissipation energy coefficient.

\section{Discussion and Application}

The stress-strain relationship can describe the deformation and failure processes of rocks, but it also has certain limitations in some aspects [38]. For example, it is difficult to accurately divide the four stages from the stress-strain curve. According to equations (4)-(7), the dissipation energy coefficient-stress curves of rocks are obtained, and three different feature points are determined as shown in (Figure 7(d)). The dissipation energy 
TABLE 2: Strength and dissipation energy coefficients at three different characteristic points.

\begin{tabular}{lccccc}
\hline \multirow{2}{*}{ Rock types } & \multicolumn{2}{c}{ Compaction point } & \multicolumn{2}{c}{ Yield point } & \multicolumn{2}{c}{ Peak point } \\
& $\sigma_{v}(\mathrm{MPa})$ & $\lambda_{v}$ & $\sigma_{u}(\mathrm{MPa})$ & $\lambda_{u}$ & $\sigma_{c}(\mathrm{MPa})$ \\
\hline Mudstone & 1.06 & 1.40 & 31.77 & 0.41 & 32.53 \\
Physicochemical slate & 1.03 & 1.31 & 31.01 & 0.39 & 32.48 \\
Schist & 1.15 & 1.76 & 34.59 & 0.31 & 41.11 \\
Limestone & 1.09 & 1.43 & 32.74 & 0.32 & 37.15 \\
Gneiss & 1.51 & 1.93 & 35.22 & 0.35 & 38.52 \\
Sandstone & 1.65 & 2.03 & 49.60 & 0.32 & 55.64 \\
Porphyrite & 1.85 & 2.23 & 55.59 & 0.29 & 0.53 \\
Dolomites & 2.89 & 2.77 & 86.70 & 0.25 & 0.53 \\
Shale & 2.81 & 2.65 & 84.44 & 0.28 & 91.57 \\
Metamorphic sandstone & 3.06 & 2.88 & 91.66 & 0.22 & 90.03 \\
Marble & 3.35 & 3.14 & 100.40 & 0.21 & 99.66 \\
Quartz schist & 4.09 & 3.58 & 122.84 & 0.17 & 0.48 \\
Quartzite & 5.23 & 4.05 & 156.79 & 0.12 & 0.35 \\
Diorite & 6.44 & 4.54 & 193.18 & 0.07 & 127.03 \\
Granite & 7.66 & 4.98 & 229.70 & 0.33 \\
\hline
\end{tabular}

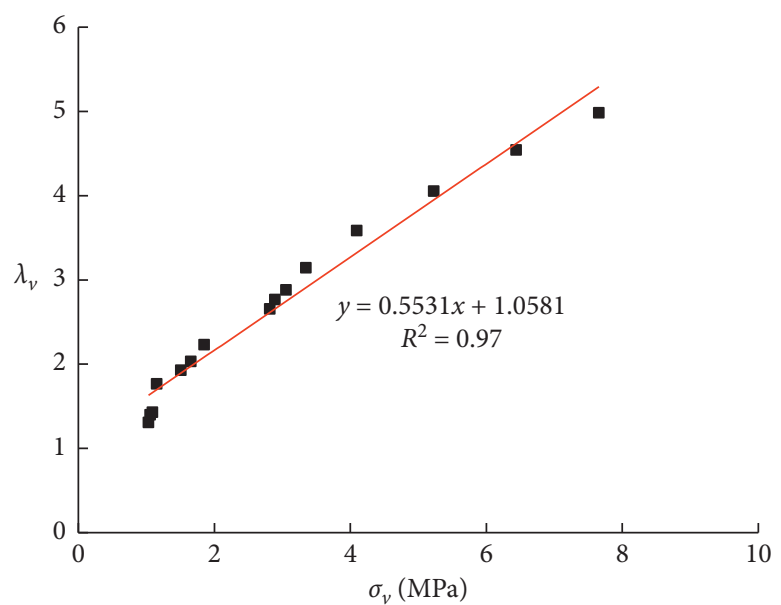

- $\lambda_{v}$ Linear fitting

(a)

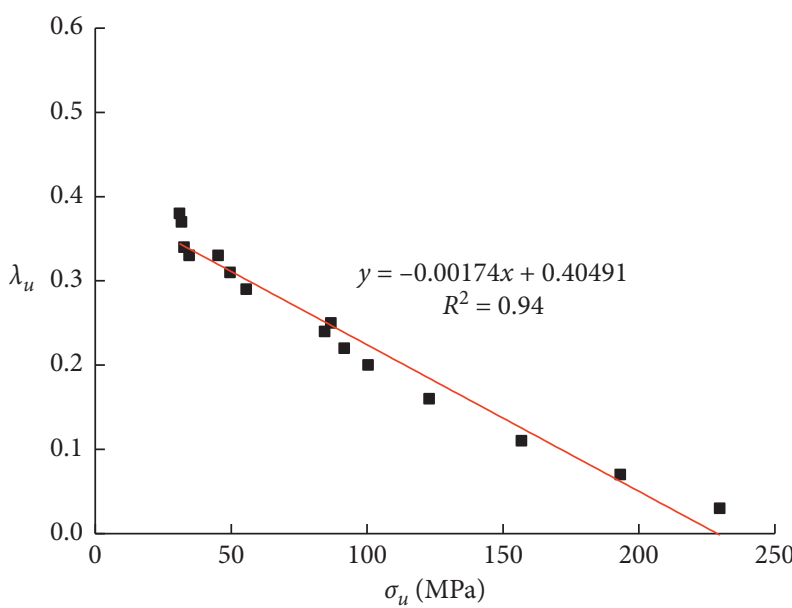

- $\lambda_{u}$

- Linear fitting

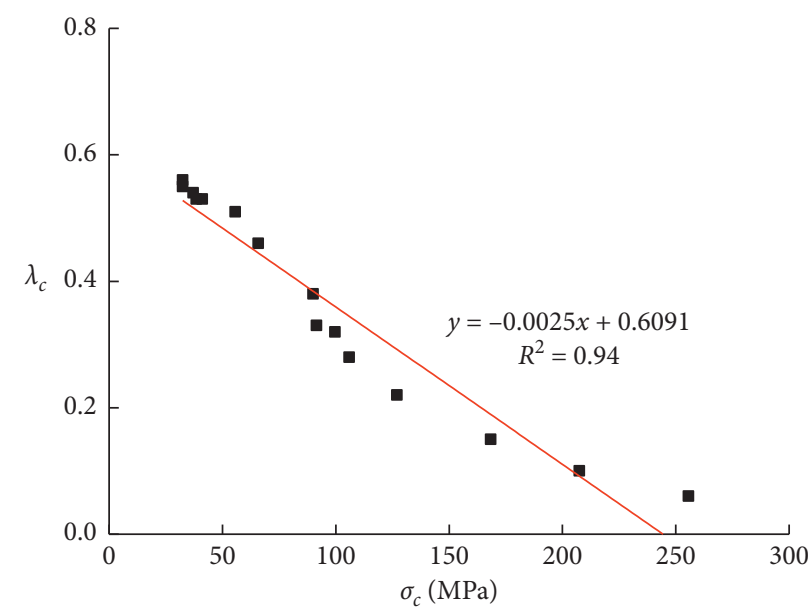

- $\lambda_{c}$

- Linear fitting

(c)

FiguRE 8: Relationships between stress and the dissipation energy coefficients at three different characteristic points. (a) Curve of the dissipation energy coefficients for the compaction point. (b) Curve of the dissipation energy coefficients for the yield point. (c) Curve of the dissipation energy coefficients for the peak point. 


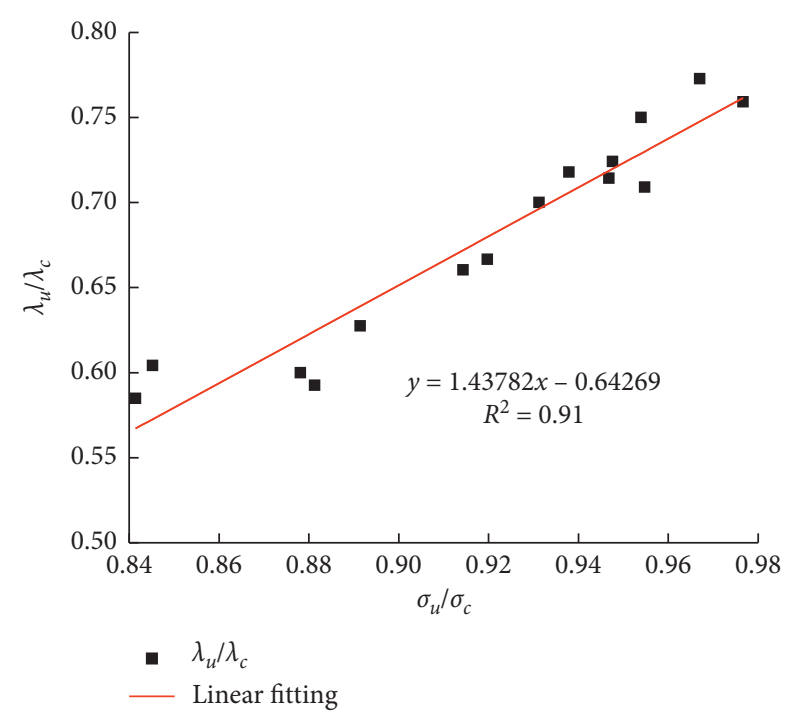

Figure 9: Relationships between the characteristic stress and the characteristic dissipation energy coefficient.

coefficient curves of rocks under uniaxial compression conditions are accurately divided into four stages by three different feature points. It approximates the $N$ shaped, corresponding to the four stages of the stress-strain curve. By comparative analysis two types of curves, the four stages of the stress-strain curve can be accurately divided (Figure $7(\mathrm{~d})$ ).

It is a difficult task to accurately determine yield strength of rocks. Usually, this value can only be approximated, which is typically $0.85 \sim 0.9$ of the peak strength [39]. The introduction of the dissipation energy coefficient provides a new idea for calculating the yield strength of the rock. The dissipation energy coefficients linearly increase with the yield strength (Figure 8(b)), and the fitting function can be expressed as follows:

$$
\lambda_{u}=-0.00174 \sigma_{u}+0.40491 .
$$

The yield strength of rocks can be obtained, which is based on equations (2)-(8) and (10).

The ratio of the total energy of the prepeak to the elastic energy at the peak is defined as the modified brittleness index [40], which can be expressed as follows:

$$
\mathrm{BIM}=\frac{U}{U^{e}},
$$

where BIM is one of the methods to determine the proneness of rock burst. The judging index is listed in Table 3 [41]. After a simple derivation, the modified brittleness index has a certain relationship with the dissipation energy coefficient:

$$
\lambda_{c}=\mathrm{BIM}-1 \text {. }
$$

A new criterion for rock burst proneness with index $\lambda_{c}$ can be proposed as follows:
TABLE 3: The judging for rock burst using the BIM value.

\begin{tabular}{lc}
\hline BIM value & Rock burst tendency \\
\hline BIM $>1.5$ & Weak rock burst \\
$1.2<\mathrm{BIM} \leq 1.5$ & Medium rock burst \\
$1.0 \leq \mathrm{BIM} \leq 1.2$ & Strong rock burst \\
\hline
\end{tabular}

$$
\left.\begin{array}{l}
0 \leq \lambda_{c} \leq 0.2 \text { Strong rockburst } \\
0.2<\lambda_{c} \leq 0.5 \text { Medium rockburst } \\
0.5<\lambda_{c} \text { Weak rockburst }
\end{array}\right\}
$$

The rock burst proneness can be divided into three categories according to a new criterion (Equation (13)). Mudstone, physicochemical slate, schist, limestone, gneiss, and sandstone are weak rock burst. Porphyrite, dolomite, shale, metamorphic sandstone, marble, and quartz schist have medium rock burst proneness. Quartzite, diorite, and granite have strong rock burst proneness. The results obtained by using the new rock burst criterion are consistent with the actual rock burst proneness.

The rate of change of the dissipation energy coefficient $(K)$ is the derivatives of the dissipation energy coefficient to the axial strain, which can be expressed as follows:

$$
K=\frac{\mathrm{d} \lambda}{\mathrm{d} \varepsilon}
$$

where $K$ is the rate of change of the dissipation energy coefficient. Figure 10 depicts the relationship between the rate of change of the dissipation energy coefficient $(K)$ and axial strain under the uniaxial compression condition. As shown in Figure 10, at the end of the compaction phase, the rate of change of the dissipation energy coefficient has a mutation $\mathrm{E}$ (the value of $K$ presents positive and negative alternating transformation). At this time, initial cracks and pores of the sample were closed. Subsequently, at the end of the elastic deformation phase, there is a mutation $\mathrm{F}$ (the value of $K$ presents negative and positive alternating transformation) in the rate of change of the dissipation energy coefficient. It indicates that new cracks begin to crack and expand. Therefore, the mutation $\mathrm{E}$ is used as the criterion for the initial crack closure of the rock, and the mutation $\mathrm{F}$ is used as the criterion for the new crack propagation of the rock.

In this paper, the conventional uniaxial compression tests for 15 different rocks were conducted. The evolution laws of the elastic properties, dissipative energy, and dissipative energy coefficient were studied. The innovation of this paper is to put forward a new calculation method of rock strength (yield strength) and to investigate the relationship between strength and the dissipation energy coefficient. Moreover, rock is a collection of minerals. The connection between mineral composition and particles is closely related to the mechanical properties and energy characteristics of rock [42]. Therefore, further work mainly focuses on the influence of the internal structure of rock on the energy 


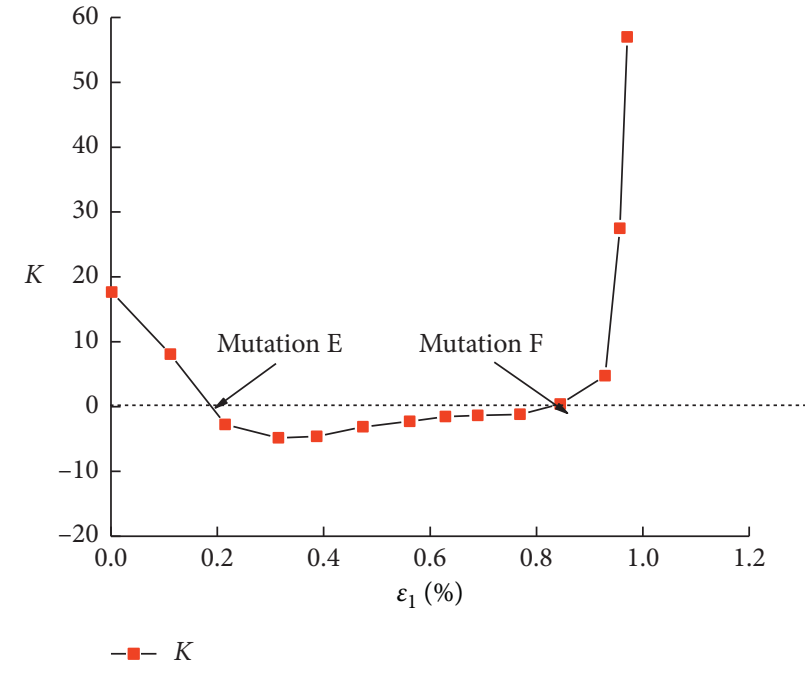

(a)

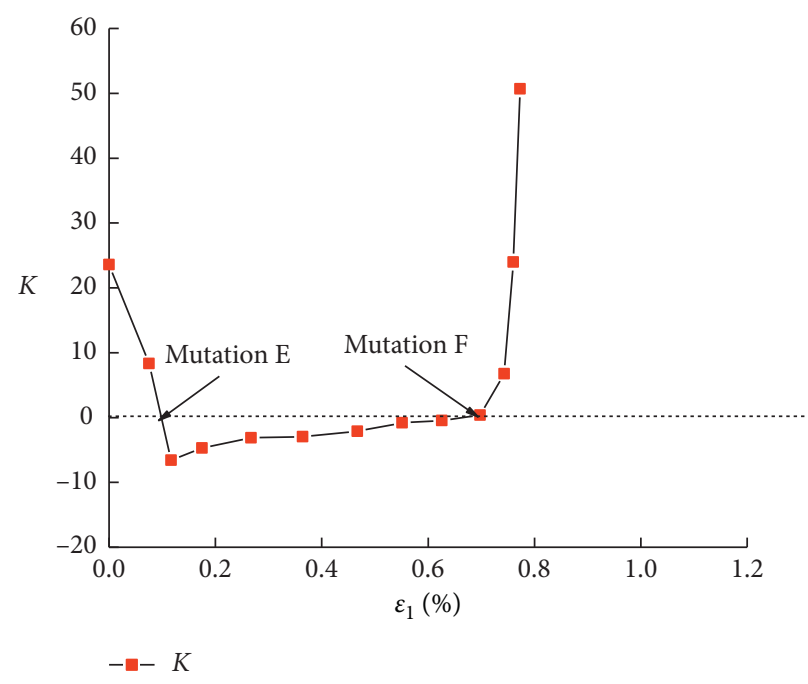

(b)

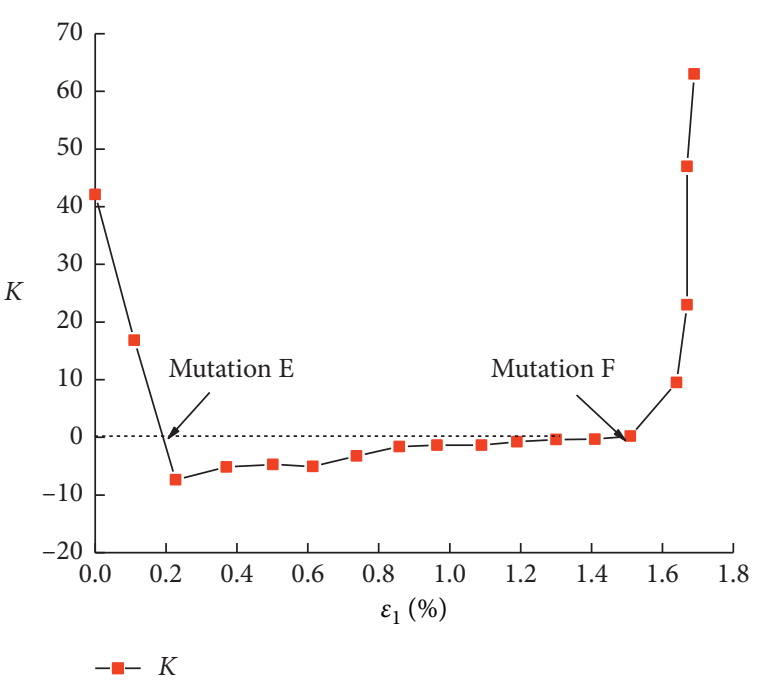

(c)

Figure 10: The curve of the rate of change of the dissipation energy coefficient (K). (a) The $K$ for marble. (b) The $K$ for shale. (c) The $K$ for sandstone.

evolution, taking into account the influence of mineral composition, fracture number, opening, fracture dip angle, trace length, and other factors, and studies the evolution law of the elastic energy, dissipative energy, and dissipative energy coefficient of rock.

\section{Conclusions}

According to the energy principle, the energy characteristics and evolution of the dissipation energy coefficient for different rocks under the uniaxial compression condition were investigated. The main conclusions are as follows:

(1) The energy evolution characteristics of different rocks are basically similar at the prepeak, but have significant differences at the postpeak.

(2) Energy evolution provides a good reflection of the rock deformation and failure process, especially the dissipation energy coefficient. It increases at first, then decreases to a minimum value, increases slowly, and finally, increases rapidly. Its evolution stage corresponds closely to the deformation stages of rocks under the uniaxial compression condition.

(3) The dissipation energy coefficients linearly increase with the compaction strength, but decrease with the yield strength and peak strength.

(4) The characteristic dissipation energy coefficients linearly increase with the characteristic stress.

(5) The dissipation energy coefficient can be used not only to accurately divide the rock deformation stage and calculate the yield strength of rocks but also as a new rock burst tendency criterion.

(6) The rate of change of the dissipation energy coefficient shows the mutation $\mathrm{E}$ and the mutation $\mathrm{F}$, respectively. The mutations indicate the initial pore closure and new crack propagation of the rock 
sample, respectively. The mutation $\mathrm{E}$ and the mutation $\mathrm{F}$ are used as the initial pore closure and new crack propagation criterion.

\section{Data Availability}

The data used to support the findings of this study are included within the article.

\section{Conflicts of Interest}

The authors declare that they have no conflicts of interest.

\section{Authors' Contributions}

MM carried out the lab work, participated in data analysis, carried out sequence alignments, participated in the design of the study, and drafted the manuscript; F Pang carried out the statistical analyses and critically revised the manuscript; $\mathrm{H}$ Wang and Y Chen collected field data and critically revised the manuscript; and J Zhu conceived the study, designed the study, and helped draft the manuscript. All authors gave final approval for publication and agreed to be held accountable for the work performed therein.

\section{Acknowledgments}

The financial support provided by the National Natural Science Foundation of China (Grants nos. 11902249 and $11872301)$ is greatly appreciated. This study is sponsored by the Natural Science Basic Research Plan in Shaanxi Province of China (2019JQ-395 and 17JS091).

\section{References}

[1] C. H. Zhu and N. Li, "Ranking of influence factors and control technologies for the postconstruction settlement of loess highfilling embankments," Computers and Geotechnics, vol. 118, 2020.

[2] M. M. He, Z. Q. Zhang, J. Ren et al., “Deep convolutional neural network for fast determination of the rockstrength parameters using drilling data," International Journal of Rock Mechanics and Mining Sciences, vol. 123, 2019.

[3] M. He, N. Li, Z. Zhang, X. Yao, Y. Chen, and C. Zhu, "An empirical method for determining the mechanical properties of jointed rock mass using drilling energy," International Journal of Rock Mechanics and Mining Sciences, vol. 116, pp. 64-74, 2019.

[4] Y. W. Li, M. Long, L. H. Zuo, W. Li, and W. C. Zhao, "Brittleness evaluation of coal based on statistical damage and energy evolution theory," Journal of Petroleum Science and Engineering, vol. 172, no. 1, pp. 753-763, 2018.

[5] F. Gong, J. Yan, S. Luo, and X. B. Li, "Investigation on the linear energy storage and dissipation laws of rock materials under uniaxial compression," Rock Mechanics and Rock Engineering, vol. 52, no. 11, pp. 4237-4255, 2019.

[6] H. P. Xie, L. Y. Li, Y. Ju, R. D. Peng, and Y. M. Yang, "Energy analysis for damage and catastrophic failure of rocks," Science China Technological Sciences, vol. 54, no. 1, pp. 192-209, 2011.

[7] Y. Ju, H. Wang, Y. Yang, Q. Hu, and R. Peng, "Numerical simulation of mechanisms of deformation, failure and energy dissipation in porous rock media subjected to wave stresses,"
Science China Technological Sciences, vol. 53, no. 4, pp. 1098-1113, 2010.

[8] X. Wang, W. Yuan, and Y. Yan, "Scale effect of mechanical properties of jointed rock mass: a numerical study based on particle flow code," Geomechanics and Engineering, vol. 21, no. 3, pp. 259-268, 2020.

[9] Q. Meng, M. Zhang, L. Han, H. Pu, and T. Nie, "Effects of acoustic emission and energy evolution of rock specimens under the uniaxial cyclic loading and unloading compression," Rock Mechanics and Rock Engineering, vol. 49, no. 10, pp. 3873-3886, 2016.

[10] Y. Wang and F. Cui, "Energy evolution mechanism in process of Sandstone failure and energy strength criterion," Journal of Applied Geophysics, vol. 154, pp. 21-28, 2018.

[11] D. Huang and Y. Li, "Conversion of strain energy in triaxial unloading tests on marble," International Journal of Rock Mechanics and Mining Sciences, vol. 66, pp. 160-168, 2014.

[12] Z. Zhang and F. Gao, "Experimental investigation on the energy evolution of dry and water-saturated red sandstones," International Journal of Mining Science and Technology, vol. 25, no. 3, pp. 383-388, 2015.

[13] Q. Ma, D. Ma, and Z. Yao, "Influence of freeze-thaw cycles on dynamic compressive strength and energy distribution of soft rock specimen," Cold Regions Science and Technology, vol. 153, pp. 10-17, 2018.

[14] T. Li, X. Pei, D. Wang, R. Huang, and H. Tang, "Nonlinear behavior and damage model for fractured rock under cyclic loading based on energy dissipation principle," Engineering Fracture Mechanics, vol. 206, pp. 330-341, 2019.

[15] H. Munoz, A. Taheri, and E. K. Chanda, "Fracture energybased brittleness index development and brittleness quantification by pre-peak strength parameters in rock uniaxial compression," Rock Mechanics and Rock Engineering, vol. 49, no. 12, pp. 4587-4606, 2016.

[16] I. R. Kivi, M. Ameri, and H. Molladavoodi, "Shale brittleness evaluation based on energy balance analysis of stress-strain curves," Journal of Petroleum Science and Engineering, vol. 167, pp. 1-19, 2018.

[17] S. Chen, D. Yin, N. Jiang, F. Wang, and Z. Zhao, "Mechanical properties of oil shale-coal composite samples," International Journal of Rock Mechanics and Mining Sciences, vol. 123, Article ID 104120, 2019.

[18] D. Li, Z. Sun, T. Xie, X. Li, and P. G. Ranjith, "Energy evolution characteristics of hard rock during triaxial failure with different loading and unloading paths," Engineering Geology, vol. 228, pp. 270-281, 2017.

[19] Y. Zhang, X. T. Feng, X. W. Zhang et al., "Strain energy evolution characteristics and mechanisms of hard rocks under true triaxial compression," Engineering Geology, vol. 260, pp. 1-14, 2019.

[20] C. E. Fairhurst and J. A. Hudson, "suggested method for the complete stress-strain curve for intact rock in uniaxial compression," International Journal of Mining Science and Technology, vol. 36, no. 3, pp. 281-289, 1999.

[21] M. He, B. Huang, C. Zhu, Y. Li, and Y. S. Chen, "Energy dissipation-based method for fatigue life prediction of rock salt," Rock Mechanics and Rock Engineering, vol. 51, no. 5, pp. 1447-1455, 2018.

[22] M. M. He, Y. S. Chen, and N. Li, "Laboratory measurements of the mechanical damping capacities in partially saturated sandstone," Arabian Journal of Geosciences, vol. 12, no. 14, pp. 1-7, 2019. 
[23] H. Xie, L. Li, R. Peng, and Y. Ju, "Energy analysis and criteria for structural failure of rocks," Journal of Rock Mechanics and Geotechnical Engineering, vol. 1, no. 1, pp. 11-20, 2009.

[24] C. Yan and B. H. Guo, "Crack closure effect and energy dissipation model for rocks under uniaxial compression," Geotechnical and Geological Engineering, vol. 38, no. 4, pp. 621-629, 2020.

[25] M. W. Zhang, Q. B. Meng, and S. D. Liu, "energy evolution characteristics and distribution laws of rock materials under triaxial cyclic loading and unloading compression," Advances in Materials Science and Engineering, vol. 228, pp. 270-281, 2017.

[26] F. Gao, S. P. Cao, K. P. Zhou, Y. Lin, and L. Y. Zhu, "Damage characteristics and energy-dissipation mechanism of frozen-thawed sandstone subjected to loading," Cold Regions Science and Technology, vol. 169, pp. 1-9, 2020.

[27] Z. Liu, C. Zhang, C. Zhang, Y. Gao, H. Zhou, and Z. Chang, "Deformation and failure characteristics and fracture evolution of cryptocrystalline basalt," Journal of Rock Mechanics and Geotechnical Engineering, vol. 11, no. 5, pp. 990-1003, 2019.

[28] Y. Zhang, N. Li, G. Lv, and Z. Chen, "Quantification of fatigue damage at dry-wet cycles of polymer fiber cement mortar," Materials Letters, vol. 257, 2019.

[29] Y. Wang, J. Tang, Z. Dai, and T. Yi, "Experimental study on mechanical properties and failure modes of low-strength rock samples containing different fissures under uniaxial compression," Engineering Fracture Mechanics, vol. 197, pp. 1-20, 2018.

[30] J. Zhao, X.-T. Feng, X.-W. Zhang, Y. Zhang, Y.-Y. Zhou, and C.-X. Yang, "Brittle-ductile transition and failure mechanism of Jinping marble under true triaxial compression," Engineering Geology, vol. 232, pp. 160-170, 2018.

[31] Q. B. Zhang and J. Zhao, "Quasi-static and dynamic fracture behaviour of rock materials: phenomena and mechanisms," International Journal of Fracture, vol. 189, no. 1, pp. 1-32, 2014.

[32] D. Ren, D. Zhou, D. Liu, F. Dong, S. Ma, and H. Huang, "Formation mechanism of the upper triassic Yanchang formation tight sandstone reservoir in ordos basin-take Chang 6 reservoir in Jiyuan oil field as an example," Journal of Petroleum Science and Engineering, vol. 178, pp. 497-505, 2019.

[33] H. Huang, T. Babadagli, X. Chen, H. Z. Li, and Y. M. Zhang, "Performance comparison of novel chemical agents for mitigating water-blocking problem in tight gas sandstones," SPE Reservoir Evaluation \& Engineering, vol. 5, no. 5, pp. 1-9, 2020.

[34] N. Zhang, W. Liu, Y. Zhang, P. Shan, and X. Shi, "Microscopic pore structure of surrounding rock for underground strategic petroleum reserve (SPR) caverns in bedded rock salt," Energies, vol. 13, no. 7, p. 1565, 2020.

[35] A. Taheri, Y. Fantidis, C. L. Olivares, B. J. Connelly, and T. J. Bastian, "Experimental study on degradation of mechanical properties of sandstone under different cyclic loadings," Geotechnical Testing Journal, vol. 39, no. 4, pp. 673-687, 2016.

[36] Y. Zhang, S. Cao, N. Zhang, and C. Zhao, "The application of short-wall block backfill mining to preserve surface water resources in northwest China," Journal of Cleaner Production, vol. 261, Article ID 121232, 2020.

[37] P. Shan and X. Lai, "An associated evaluation methodology of initial stress level of coal-rock masses in steeply inclined coal seams, Urumchi coal field, China," Engineering Computations, vol. 37, no. 6, pp. 2177-2192, 2020.
[38] S.-Q. Yang, "Experimental study on deformation, peak strength and crack damage behavior of hollow sandstone under conventional triaxial compression," Engineering $\mathrm{Ge}$ ology, vol. 213, pp. 11-24, 2016.

[39] M. He, Z. Zhang, J. Zheng, F. Chen, and N. Li, "A new perspective on the constant mi of the Hoek-Brown failure criterion and a new model for determining the residual strength of rock," Rock Mechanics and Rock Engineering, vol. 53, no. 9, pp. 3953-3967, 2020.

[40] M. Aubertin, D. E. Gill, and R. Simon, "On the use of the brittleness index modified (BIM) to estimate the post-peak behavior or rocks," Aqua Fennica, vol. 232, pp. 4-25, 1994.

[41] M. Cai, "Prediction and prevention of rockburst in metal mines-a case study of Sanshandao gold mine," Journal of Rock Mechanics and Geotechnical Engineering, vol. 8, no. 2, pp. 204-211, 2016.

[42] W. He, K. Chen, A. Hayatdavoudi, K. Sawant, and M. Lomas, "Effects of clay content, cement and mineral composition characteristics on sandstone rock strength and deformability behaviors," Journal of Petroleum Science and Engineering, vol. 176, pp. 962-969, 2019. 